\title{
Correction to: identification of multiple cancer-associated myositis-specific autoantibodies in idiopathic inflammatory myopathies: a large longitudinal cohort study
}

Hanbo Yang ${ }^{1,2+}$, Qinglin Peng ${ }^{1 \dagger}$, Liguo Yin ${ }^{1}$, Shanshan Li ${ }^{1}$, Jingli Shi ${ }^{1}$, Yamei Zhang ${ }^{1}$, Xin Lu ${ }^{1}$, Xiaoming Shu ${ }^{1}$, Sigong Zhang ${ }^{3}$ and Guochun Wang ${ }^{1,2^{*}}$

\section{Correction}

After publication of the article [1], it has been brought to our attention that the labels in Fig. $2 b$ have been switched and are as a result incorrect. The label for the red line should have the label "non-CAM" and the yellow line "CAM".

\section{Author details \\ 'Department of Rheumatology, Beijing Key Lab for Immune-Mediated Inflammatory Diseases, China-Japan Friendship Hospital, 2 Yinhua Road, Chaoyang District, Beijing 100029, China. ${ }^{2}$ Graduate School of Peking Union Medical College, Beijing 100730, China. ${ }^{3}$ Department of Rheumatology, Lanzhou University Second Hospital, Gansu province 730046, China.}

Received: 5 March 2018 Accepted: 6 March 2018

Published online: 11 April 2018

\section{Reference}

1. Yang H, Peng Q, Yin L, Li S, Shi J, Zhang Y, et al. Identification of multiple cancer-associated myositis-specific autoantibodies in

idiopathic inflammatory myopathies: a large longitudinal cohort study. Arthritis Res Ther. 2017;19:1. https://doi.org/10.1186/s13075-017-1469-8.

\footnotetext{
* Correspondence: guochunwang@hotmail.com

${ }^{\dagger}$ Equal contributors

${ }^{1}$ Department of Rheumatology, Beijing Key Lab for Immune-Mediated Inflammatory Diseases, China-Japan Friendship Hospital, 2 Yinhua Road, Chaoyang District, Beijing 100029, China

${ }^{2}$ Graduate School of Peking Union Medical College, Beijing 100730, China
} 